\title{
Uncertainty about uncertainty within a stakeholder group
}

\author{
$\underline{\text { T. Chan }}^{\text {a,b }}$, P. McShane ${ }^{\text {a }}$ and H. Ross ${ }^{c}$ \\ ${ }^{a}$ Monash Sustainability Institute, Monash University, Victoria \\ ${ }^{b}$ Water Studies Centre, Monash University, Victoria \\ ${ }^{c}$ School of Integrative Systems, University of Queensland, Queensland \\ Email: terence.chan@monash.edu
}

\begin{abstract}
The explicit quantification of uncertainty is one of the useful features of Bayesian network models. Identification and quantification of uncertainty is particularly important in complex fields such as environmental management, where there may be great natural variability and data uncertainty, and there may not be sufficient data available to characterise all relevant variables. Bayesian networks also encourage explicit decisions about the subjective choices that can be common in management generally, for example in deciding on the relative importance of non-comparable variables.

In this paper, some of the data from a previous study in the Bayesian framework is further examined. The previous project is described in more detail in a previous paper, however, here we focus specifically and in more detail on the expert elicitation process, outcomes and implications than was possible in the previous paper. The original project involved using an Ecological Risk Assessment approach to guide the overall project design, with a participatory approach used to build a Bayesian network model with input from a range of stakeholders in the Kongulai Catchment in the Solomon Islands. This process incidentally produced quantified subjective opinion data from one group of expert stakeholders, those involved professionally in water or catchment management.
\end{abstract}

Specifically, we examine the variation in information elicited from the individual experts for the conditional probability tables used in the Bayesian network model, using Median Absolute Deviation (MAD) as a simple but robust descriptive statistic to quantify differences in opinion between experts. For univariate data $X_{1}, X_{2}$, $\ldots \mathrm{X}_{\mathrm{n}}$; the $\mathrm{MAD}=\operatorname{median}_{\mathrm{i}}\left(\left|\mathrm{X}_{\mathrm{i}}-\operatorname{median}_{\mathrm{j}}\left(\mathrm{X}_{\mathrm{j}}\right)\right|\right)$. That is, the median of the set of absolute differences between each value and the median.

The quantification of variation in opinion within this group allows us to explore and identify sources of this variation and divergence. As the experts provided estimates of uncertainty regarding catchment relationships, the MAD then provides a measure of their 'uncertainty about uncertainty'. Some primary features illustrated with this representation of variation in expert opinion:

- the greatest agreement between experts (lowest MAD) usually occurs where pressures (parent variables) all act on a variable in the same direction,

- greater disagreement (higher MADs) are seen where pressures act on a variable in opposing directions, and also where there is one or several variables where little is known,

- the highest average MAD can be used to identify what combination of pressures cause the greatest uncertainty between experts, and can narrow identification where there is one of multiple pressures causing the greatest uncertainty.

Difficulties encountered in the use of this measure include its low resolution and discarded outliers, inconsistencies in expert opinion, and time constraints limiting the complexity of the elicitation and the number of experts involved. Further work would be valuable to explore the impact of a larger group of experts, including those with non-traditional forms of expertise, such as local knowledge, and alternative elicitation processes. How quantification of uncertainty, and further, our uncertainty about uncertainty, can be used in the wider context of natural resource management remains unresolved.

Keywords: Uncertainty, Bayesian network model, catchment management, expert elicitation, expert opinion 


\section{INTRODUCTION}

In this paper some of the data from a previous study in the Bayesian framework is further examined. The previous project is described in detail in Chan et al. (2010), however, here we focus specifically and in more detail on the expert elicitation process, outcomes and implications than was possible in the previous paper.

The complexity of environmental systems ensures that in environmental modelling, there are always numerous unknown or unrecognised cause-effect relationships (Harris 1994, Townsend and Riley 1999). Additionally, unless a particular site has been the focus of long-term research, there is unlikely to be a significant history of quantitative data collected and analysed to parameterise the known relationships (Likens 1989, Carpenter 2002, Letcher et al. 2002). It is thus sometimes not possible to use traditional mechanistic, process-based modelling approaches to simulate integrated environmental systems in the detail desirable for management. This deficiency has resulted in an increase in the use of tools such as Bayesian network models, which can use 'experts' to help define the unknowns for modelling (Korb and Nicholson 2004, Martin et al. 2005, Hart et al. 2006). Studies have also found expert input to be valuable in moderating field data and in narrowing prediction intervals, particularly under data-poor conditions (Martin et al. 2005).

There is inherent imprecision in all models and also in expert input to models, as well as in the actual process of extracting information from experts (O'Hagan and Oakley 2004). A number of studies look at how best to conduct elicitation, and problems associated with this imperfect process (Kadane and Wolfson 1998, O'Hagan and Oakley 2004, Mac Nally 2007, Kynn 2008). Expert elicitation in the specific Bayesian framework is also increasingly studied in recent years (e.g. Cain 2001, Martin et al. 2005, James et al. 2010).

Bayesian network methods are particularly appropriate in environmental contexts, given not just sparse data (requiring the use of expert opinion), and the need to communicate results to non-specialists for management decision-making (addressed by the use of a graphical interface), but also due to high uncertainty from multiple sources (Ellison 1996). Uncertainty can be defined as "any departure of the unachievable ideal of complete determinism" and can be due to 1. natural variability and indeterminism, or 2. lack of knowledge/understanding (Walker et al. 2003). In our case, we are primarily concerned with the second type of uncertainty, and in particular, the 'uncertainty about uncertainty' associated with elicitation of probability estimates from experts.

\section{METHODS}

An Ecological Risk Assessment approach was used to guide the overall project design (Hart et al. 2006). A participatory approach was used to build a Bayesian network (BN) model. BNs were selected as a relatively intuitive form of modelling tool (due to their graphical interface), and there was specific interest in developing capacity and understanding in this area from our in-country partners. Our ability to further use the data from the model development process to explore consensus in this paper was incidental (and fortuitous). Detailed methodology is available in Chan et al. (2010), but a summary of key parts is provided below, with additional methods appropriate for our focus on, and analysis of the expert input used in this case.

\subsection{Software}

The software used to build the Bayesian network in both case studies is called Netica ${ }^{\mathrm{TM}}$, and is available from http://www.norsys.com (Norsys 2005). A detailed background to Bayesian networks and modelling is available from Korb and Nicholson (2004).

\subsection{Study Site}

The study site was the Kongulai Catchment on the island of Guadalcanal in the Solomon Islands (Chan et al. 2010). The objective of the original project was to research ways to assist in local implementation of integrated catchment management for water resources. This catchment extends west and south from the national capital, Honiara, covering approximately $50 \mathrm{~km}^{2}$, with about $1 \mathrm{~km}^{2}$ coinciding with the city itself, the remaining area being under customary (traditional) ownership. The terrain is primarily mountainous and covered with heavy tropical forest, with little road access. There are few people inhabiting the bulk of the catchment, although informal settlements exist in a narrow strip along the coastal road, with accompanying subsistence agriculture. A very small part of the catchment is leased by the Solomon Islands Water Authority (SIWA) from the traditional owners, and an impoundment collects water from a spring, which is then pumped to water storages for the city. There was little quantitative data available apart from water quality measurements, scattered in time and space (Chan et al. 2010). 


\subsection{Expert elicitation}

A BN was produced from the input of a variety of stakeholders, with longer term, more focused input from a smaller number of experts. These inputs included an initial system conceptualisation for development of the model structure, followed by quantitative definition of variable relationships by estimating conditional probabilities (i.e. defining Conditional Probability Tables or CPTs). Quantitative development of the model was restricted to government representatives, all working in water management or policy. The experts developed CPTs independently, and these were then averaged for the final (prior) CPTs. The model also had additional quantitative (field) data inputs used to train the averaged CPT relationships elicited, according to Lauritzen and Spiegelhalter (1990). The results from this process were presented to all stakeholder groups for feedback and discussion. Model behaviour after CPT averaging and quantitative data training is described in Chan et al. (2010). In this paper, however, we are concerned with the originally elicited expert CPTs.

For this study, we examine the variation in information elicited from the individual experts for the CPTs, using Median Absolute Deviation (MAD) as a very simple descriptive statistic to quantify differences in opinion between experts. For univariate data $X_{1}, X_{2}, \ldots X_{n}$; the MAD $=\operatorname{median}_{i}\left(\left|X_{i}-\operatorname{median}_{j}\left(X_{j}\right)\right|\right)$. That is, the median of the set of absolute differences between each value and the median (Pham-Gia and Hung 2001).

The MAD is used as a simple and robust measure of statistical dispersion, and is particularly useful in this case (with limited data) as there is less influence from 'outliers' (Pham-Gia and Hung 2001, Mac Nally 2007). In this case, the MAD may also be regarded as a measure of uncertainty about uncertainty, as the experts are estimating the uncertainty (probability) of a variable being in a particular state. A smaller MAD thus indicates greater agreement between experts. Note that mean and standard deviations over expert outputs (e.g. Mac Nally 2007), or a measure of entropy (e.g. Lemos 2010) could also have been used as measures of variability, however the limited number of experts and the resulting greater influence of outliers, as well as the discretised nature of the elicited uncertainty estimates (to a resolution of $5 \%$ certainty) made these measures less useful.

It should also be noted that CPTs were only elicited for those variables where the experts felt some confidence in their knowledge. Other CPTs were collaborated on, and still other variables were left with a uniform probability distribution, reflecting the perceived lack of knowledge (e.g. particularly where variables were to do with governance or cultural issues). Where CPTs were elicited, there were also a limited number of experts (six to eight). However, as there remain many individually elicited CPTs (17), and as each CPT is a matrix (sometimes quite large), only key trends and illustrative results are reported here.

\section{RESULTS}

\subsection{Quantified variation in expert opinion - uncertainty about uncertainty}

As an illustration of the process of quantifying variation amongst expert opinion via their elicited CPTs, Figure 1 shows part of the larger Bayesian network model developed for this catchment (the full BN may be seen in Chan et al., 2010). This branch shows the 'parent' variables considered by the stakeholders to be the primary influences (erosion and flooding) on sediment in the catchment runoff (which eventually provides the water supply). Table 1 shows the layout of the CPTs elicited for this variable, with entries showing the range of probabilities given by the group of experts. Table 2 shows the associated median CPT values and their MADs. It can be seen here that where a variable of concern has only two potential states, the MAD is uniform across states, this starts to vary when there are more

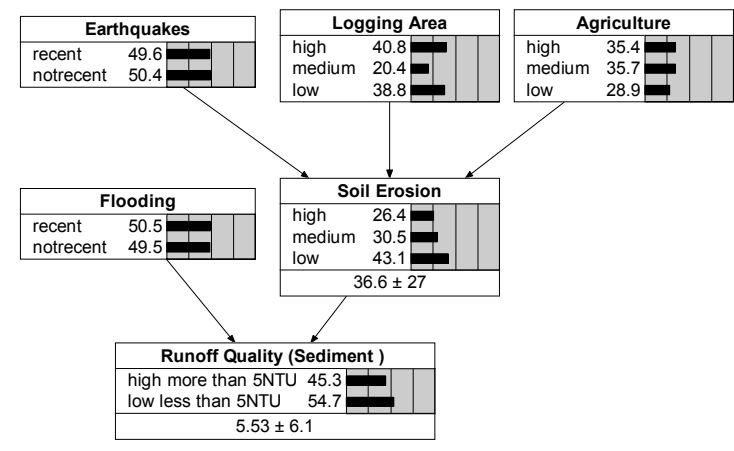

Figure 1. Part of one branch of the Bayesian network model for the Kongulai catchment. potential states, as seen in Tables 3 and 4.

However, as higher variation in any one of these elicited conditional probabilities will necessarily be reflected in the complementary probabilities, each row of the CPT can be averaged across states and retain a meaningful deviation relative to the other rows in a table (e.g. compare the average/mean MAD in the last columns of Tables 3 and 4 with the individual MADs). Table 3 summarises an additional set of medians and 
MADs for the CPTs for soil erosion with three parent variables: (minor) earthquakes, logging and agriculture. Table 4 summarises the variation in how experts perceived the water quantity available for human use being a product of four parent variables. Some primary features are clearly illustrated with this representation of variation in expert opinion.

1. The greatest agreement usually occurs where pressures (parent variables) all act on a variable in the same direction. E.g. where both Flooding and Soil Erosion occur or are 'high' (top row of Tables 1 and 2), the MAD is zero (top row of Table 2) and the experts have high agreement about how likely it is that sediment will be high in the runoff. Similarly, it can be seen that there is high agreement about when there is likely to be low sediment in runoff (bottom row of Tables 1 and 2). The same pattern is seen in Tables 3 and 4 and also in other CPTs not shown here, such as Runoff Quality (Microbes), Supply Quality (Sediment), Supply Quality (Microbes), Household water supply dependability, Logging, etc.

2. In many cases (particularly where there are only two parent variables), the greatest variation (dispersion) usually occurs where the state of parent variables cause opposing pressures on the child variable, e.g. in Table 2, Runoff Quality (Sediment) has the highest MAD and the most disagreement between experts when the two primary influential variables are in opposition. That is, when there is flooding but no soil erosion, or soil erosion, but no flooding (middle rows).

Table 1. Runoff Quality (Sediment) CPT: range of original expert elicited probabilities

\begin{tabular}{cccc}
\hline Flooding & $\begin{array}{c}\text { Soil } \\
\text { Erosion }\end{array}$ & $\begin{array}{c}\text { Likelihood that Runoff Quality } \\
\text { (Sediment) is: } \\
\text { High }(>5 \mathrm{NTU})\end{array}$ & Low $(<5 \mathrm{NTU})$ \\
\hline Yes & High & $1.00 \ldots 0.70$ & $0.00 \ldots 0.30$ \\
Yes & Medium & $0.90 \ldots 0.50$ & $0.10 \ldots 0.50$ \\
Yes & Low & $0.65 \ldots 0.20$ & $0.35 \ldots 0.80$ \\
No & High & $0.15 \ldots 0.50$ & $0.85 \ldots 0.50$ \\
No & Medium & $0.05 \ldots 0.30$ & $0.95 \ldots 0.70$ \\
No & Low & $0.00 \ldots 0.10$ & $1.00 \ldots 0.90$ \\
\hline
\end{tabular}

Table 2. Medians and MADs for Runoff Quality (Sediment).

\begin{tabular}{cccccc}
\hline \multirow{2}{*}{ Flooding } & Soil Erosion & \multicolumn{3}{c}{ Munoff Quality (Sediment) } \\
& & High & Low & High & Low \\
\hline Yes & High & 1.00 & 0.00 & 0.00 & 0.00 \\
Yes & Medium & 0.80 & 0.20 & 0.10 & 0.10 \\
Yes & Low & 0.50 & 0.50 & 0.15 & 0.15 \\
No & High & 0.40 & 0.60 & 0.10 & 0.10 \\
No & Medium & 0.25 & 0.75 & 0.05 & 0.05 \\
No & Low & 0.00 & 1.00 & 0.00 & 0.00 \\
\hline
\end{tabular}

3. Less trivially than the above points, where there are more than two parent variables (e.g. Tables 3 and 4), the highest average/mean MAD can also be used to identify the more complex situations causing uncertainty between experts. In Table 3 , the greatest uncertainty surrounded the amount of erosion that occurs when there was minimal disturbance from logging, agriculture and the regular tremors that occur in the catchment (the last two of the key rows shown).

Table 3. Medians and MADs for "Soil erosion" (key rows only).

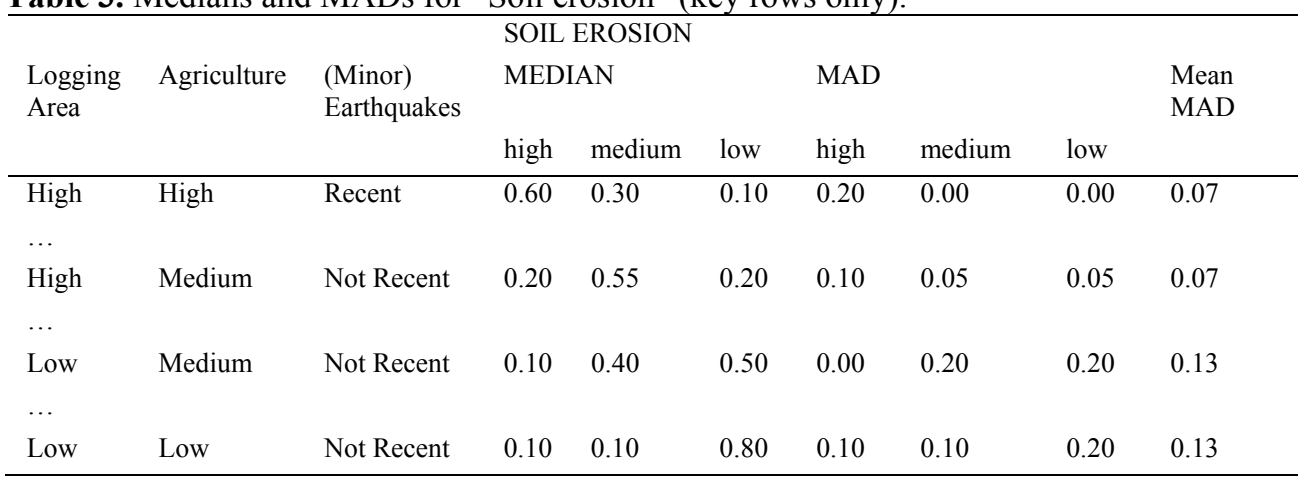

4. The highest MAD can be used to identify which of the parent variables is the source of greatest uncertainty between experts. In Table 4, the highest MADs occur when leakage is high, rather than when the three other parent variables are in a restrictive state. For example, we can compare the impact of leakage vs access (to piped water with a regular supply) by looking at the MADs in row 2 vs that in row 1 and row 3 . This is further shown by the highest individual MAD ( 0.25 on row 6) occurring when experts were asked to consider the likelihood that inadequate water is supplied, where all the parent variables are in states that should provide adequate water, except for 'leakage'. 
Chan et al., Uncertainty about uncertainty within a stakeholder group

Table 4. Water Quantity (for domestic use) expert elicited CPT, median, MAD, average MAD (key rows).

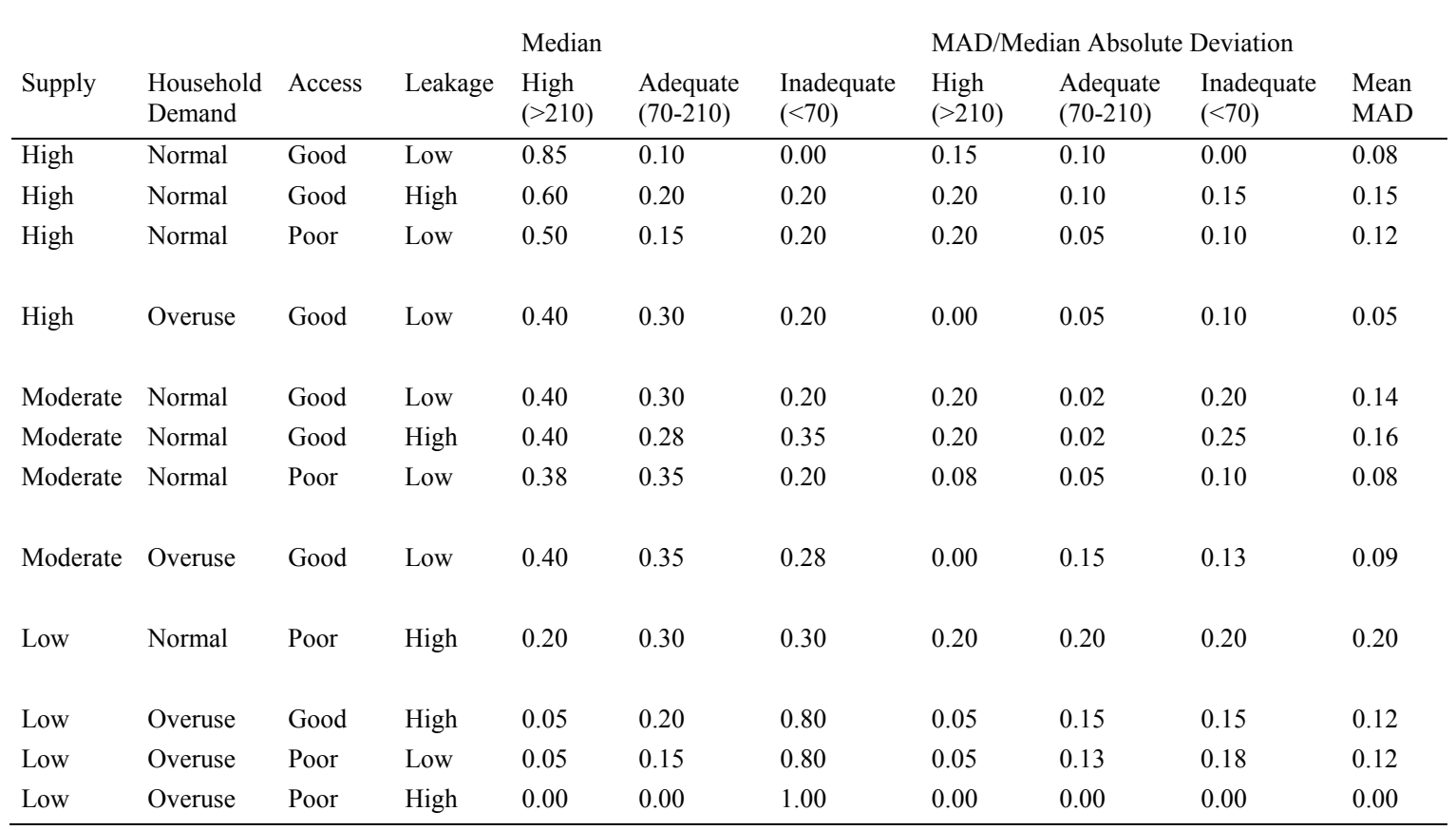

\section{DISCUSSION}

Although the MAD reveals only trivial features in the relatively simple cases (e.g. in Table 2), where variable relationships become more complex, the MAD begins to allow more interesting identification of where the greatest divergence of expert opinion occurs, and thus which factors and relationships may be at issue. The additional complexity is reflected in an increase in the size of CPT under consideration for a variable - this may be due to a larger number of parent variables, or a larger number of states in the parent or child variables. However, even with the MAD, determining where the greatest disagreement amongst experts occurs may not be straightforward. In particular, the experts may not be consistent with their estimations throughout a single CPT (particularly where these are complex), potentially due to natural human biases arising from framing and human cognitive processes (Kynn 2008).

The method of expert elicitation for population of a Bayesian network presented here was relatively simple and direct. More complex forms of elicitation for detailed distributions (such as the definition of probability density functions) have also been developed, with various tools intended to make the required elicitation easier, and account for human bias (e.g. specialised software). These tools also have the potential to instantly highlight inconsistencies within a CPT for revision, and enable direct analysis of variation in expert opinion (Al-Awadhi and Garthwaite 1998, James et al. 2010). However, these tools may not be practical in many cases, because of time, computing resources, or because of the experts' training and background (O'Hagan and Oakley 2004). The direct elicitation used in this case can attempt to minimise inconsistencies with highly structured processes to check for inconsistencies in CPTs and provide iterative opportunities to rectify them.

Note that the robustness of the MAD measure also results in (i.e. requires some) coarseness, particularly where there are few experts (as in this case study). Discarded outlying probabilities can represent a significant proportion of the expertise available, despite these outliers being evidence of some variation in expert opinion from the median. As an example, in the first row of Table 1 it can be seen that there was some variation in the expert elicited probabilities, but because most of the experts agreed on the same probability distribution, two deviations from this were discarded by the MAD calculation, and Table 2 shows a MAD of zero in the first row.

A related facet of the coarseness of the MAD measure is that given the discrete distributions used in the Bayesian network, the experts are giving approximations of uncertainty over the range of each state (e.g. in Table 1, experts are estimating the likelihood that sediment in runoff quality is "more than 5 NTU"). Additionally, in this study, the experts were understandably not comfortable estimating likelihood to a finer than $5 \%$ resolution. Even this resolution may be finer than information and understanding can realistically provide, and elicitation of uncertainties to the closest $10 \%$ may be the highest level of precision that can be expected (Cain et al. 2001). 
A larger sample of experts would be valuable in further examining the usefulness of the MAD. One potential source for additional data was from the larger pool of stakeholders. Few of the stakeholders outside the expert group had much experience in quantitative matters, however, local, place-based, experiential expertise of the community landowners could provide key input (e.g. Fazey et al. 2006). The traditional landowners had much more frequent and widespread access to the catchment, and were able to provide information about the location and behaviour of sinks and springs in the karst geology, the impacts of landslides and the regular minor earthquakes on water flow through the catchment and about the occurrence of logging. Further investigation is needed into how to ensure that inclusion of local knowledge is reliable (e.g. via additional monitoring/sampling to validate data, via set up of systematic approaches where local volunteers are involved, or other methods), however there is potential to elicit basic likelihood estimates and CPTs, for example, in terms of the frequency and severity of earthquakes, and the history and extent of logging, from these non-traditional "experts", although (as in this case) time constraints may make this infeasible.

Similarly, in the case of interdisciplinary, integrated modelling efforts, time constraints often limit elicitations to those which are relatively simple and limited across the range of important processes, in comparison to other projects which may focus on a single process (e.g. Mac Nally 2007, which has a very detailed analysis of the expert input into predicting the breeding success of a specific bird species).

An additional issue requiring further research particularly relevant to this case, but not yet explored, is the non-independence of experts consulted. Even given a large group of experts, the experts will often have common factors compromising their apparent independence, including the limited number of people with a high level of knowledge in any particular specialised topic, the number of potential people who may know a specific system (such as a catchment) very well, similar backgrounds, whether educational/academic or workplace-based, and employment at the same agencies and departments. Convergence of elicited information may thus be more of a measure of shared background rather than certainty in the processes under consideration. It should also be reiterated that as used in this paper, the MAD is specifically a measure of agreement among experts, rather than a measure of uncertainty about the data available, although variability or uncertainty about the data may be one of the reasons leading to agreement or disagreement among the experts.

Finally, it should be noted that although this paper focuses solely on the role of expert input, and although the role of expert opinion will remain vital in situations where data is limited, such as in developing countries and remote or inaccessible locations (Howard et al. 2006), there is the broader context of management to be considered. Field monitoring, cyclical validation against the model outputs, and iterative improvement of models are the way in which expert systems and decisions are improved, and are essential to closing the adaptive management process cycle.

\section{CONCLUSIONS}

A successful participatory process enabled construction of a Bayesian network model for the Kongulai Catchment, aimed at supporting decision making by on-ground water and catchment managers. A key part of this process was eliciting quantified expert opinion about many relationships within the catchment system for which there was little data. Analysis of the variation between expert opinions is illustrated as useful for identifying situations of greatest consensus as well as greatest uncertainty, and the median absolute deviation provides a measure of the uncertainty about uncertainty. Additional attention to the situations where this uncertainty about uncertainty is greatest, for example, by the managers and through additional monitoring, would enable reduction of this uncertainty. However, a limited pool of experts can result restrict the usefulness of this analysis. Wider representation and broader definitions of expertise that encompass local knowledge and use of participatory processes are of value in reducing uncertainty about data used in modelling as well as for the additional social learning benefits and potential for improving environmental management, particularly in developing countries where formally collected data can be scarce.

Additionally, expert elicitation is difficult, and can be fraught by inconsistency, bias, and practical limitations. Efforts to remedy these problems can take the form of specialised software or structured iterative expert participation and elicitation techniques. Variations in expert opinion can still yield useful outcomes, and expert opinion will continue to be needed into the future as long as data is inadequate. However, there remain a number of areas where further research into expert elicitation and input is needed. It also remains unresolved how we might best use quantification of uncertainty, and further, uncertainty about uncertainty, within the wider context of natural resource management. 


\section{ACKNOWLEDGMENTS}

The Solomon Islands case study was funded by AusAID and performed in collaboration with the International WaterCentre, the Solomon Islands Water Authority and the Division of Water Resources in the Solomon Islands Department of Mines and Energy. We thank the original research team: Bronwyn Powell and Suzanne Hoverman, and the local liaisons, experts and stakeholders who so kindly provided their time.

\section{REFERENCES}

Al-Awadhi, S.A. and P.H. Garthwaite. (1998). An elicitation method for multivariate normal distributions. Communications in Statistics-Theory and Methods, 27, 1123-1142.

Cain, J. (2001). Planning improvements in natural resource management: Guidelines for using Bayesian networks to support the planning and management of development programmes in the water sector and beyond. Centre for Ecology and Hydrology, Crowmarsh Gifford, Wallingford, Oxon.

Carpenter, S.R. (2002). Ecological futures: Building an ecology of the long now. Ecology, 83, 2069-2083.

Chan, T., H. Ross, S. Hoverman, and B. Powell. (2010). Participatory development of a Bayesian network model for catchment-based water resource management. Water Resources Research, 46, W07544.

Ellison, A. M. (1996). An Introduction to Bayesian Inference for Ecological Research and Environmental Decision-Making. Ecological Applications, 6, 1036-1046.

Fazey, I., J.A. Fazey, J.G. Salisbury, D.B. Lindenmayer, and S. Dovers. (2006). The nature and role of experiential knowledge for environmental conservation. Environmental Conservation, 33, 1-10.

Harris, G.P. (1994). Pattern, Process and Prediction in Aquatic Ecology - a Limnological View of Some General Ecological Problems. Freshwater Biology, 32, 143-160.

Hart, B. T., Burgman, M., Grace, M., Pollino, C., Thomas, C., Webb, J. A. (2006). Risk-based approaches to managing contaminants in catchments. Human and Ecological Risk Assessment, 12, 66-73.

Howard, G., S. Pedley, and S. Tibatemwa. (2006). Quantitative microbial risk assessment to estimate health risks attributable to water supply: can the technique be applied in developing countries with limited data? Journal Of Water And Health, 4, 49-65.

James, A., S.L. Choy, and K. Mengersen. (2010). Elicitator: An expert elicitation tool for regression in ecology. Environmental Modelling \& Software, 25, 129-145.

Kadane, J.B. and L.J. Wolfson. (1998). Experiences in elicitation. Journal of the Royal Statistical Society Series D-the Statistician, 47: 3-19.

Korb, K.B. and A.E. Nicholson. (2004). Bayesian Artificial Intelligence. Chapman and Hall/CRC Press, London.

Kynn, M. (2008). The 'heuristics and biases' bias in expert elicitation. Journal of the Royal Statistical Society Series a-Statistics in Society, 171: 239-264.

Lauritzen, S.L. and D.J. Spiegelhalter. (1990). Local computations with probabilities on graphical structures and their application to expert systems. Pages 415-458. in G. Shafer and J. Pearl, editors. Readings in Uncertain Reasoning. Morgan Kauffman.

Lemos, M.C., A.R. Bell, N.L. Engle, R.M. Formiga-Johnsson, and D.R. Nelson. (2010). Technical knowledge and water resources management: A comparative study of river basin councils, Brazil. Water Resources Research, 46: W06523.

Letcher, R.A., A.J. Jakeman, M. Calfas, S. Linforth, B. Baginska, and I. Lawrence. (2002). A comparison of catchment water quality models and direct estimation techniques. Environmental Modelling \& Software, $17,77-85$.

Likens, G.E., editor. (1989). Long-term studies in ecology: approaches and alternatives. Springer-Verlag New York; Berlin.

Mac Nally, R. (2007). Consensus weightings of evidence for inferring breeding success in broad-scale bird studies. Austral Ecology, 32, 479-484.

Martin, T.G., P.M. Kuhnert, K. Mengersen, and H.P. Possingham. (2005). The power of expert opinion in ecological models using Bayesian methods: Impact of grazing on birds. Ecological Applications, 15, 266280.

Norsys. 2005. Netica. http://www.norsys.com

O'Hagan, A. and J.E. Oakley. (2004). Probability is perfect, but we can't elicit it perfectly. Reliability Engineering \& System Safety, 85, 239-248.

Pham-Gia, T. and T.L. Hung. (2001). The mean and median absolute deviations. Mathematical and Computer Modelling, 34, 921-936.

Walker, W.E., P. Harremoes, J. Rotmans, J.P. van der Sluijs, M.B. A. van Asselt, P. Janssen, and M.P.K. von Krauss. (2003). Defining Uncertainty: A Conceptual Basis for Uncertainty Management in Model-Based Decision Support. Integrated Assessment, 4, 5-17. 